\section{Vignola joins SESAME}

Gaetano Vignola, well known in the synchrotron radiation accelerator community, joined SESAME as the machine director. $\mathrm{He}$ began his career in accelerator physics in 1967 when he joined the ADONE group in Frascati National Laboratories, where he was involved also in the commissioning of a $2 \mathrm{~T}$ wiggler and in freeelectron laser experiments. In 1983 he moved to the NSLS at Brookhaven, where he participated actively in the commissioning of the facility. In 1990 he went back to Frascati where he proposed and successfully led the construction of the DAFNE F-factory, a highluminosity high-current $1 \mathrm{GeV}$ collider.

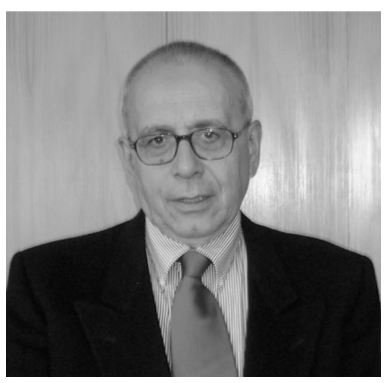

Gaetano Vignola.

\section{APS Rosalind Franklin Young Investigator Award}

Dr Alexis S. Templeton has been chosen to receive the first APS Rosalind Franklin Young Investigator Award. Her work as a graduate student at Stanford University and more recent studies as a Postdoctoral Research Associate at Scripps Institution of Oceanography, University of California, San Diego, centres around the influence of microorganisms in the speciation of heavy metals in environmental systems, as well as the role of bacteria in the weathering of basaltic glasses in deep ocean environments. To simultaneously investigate both the abiotic and biologically-mediated reactions at biofilm/mineral interfaces, Alexis has relied heavily on a diverse array of spectroscopic and microscopic techniques. For her graduate work, she used microbeam X-ray fluorescence, conventional and grazing-angle $\mathrm{X}$-ray absorption spectroscopy, and long-period $\mathrm{X}$-ray standing wave (XSW) synchrotron-based techniques. A particularly novel development was her combination of the XSW technique with XANES spectroscopy to determine the vertical distribution and speciation of selenium within microbial biofilms formed on oxide surfaces. The combined methods she used provide a fully three-dimensional characterization of trace-element distribution and speciation at a complex interface and represent a major advance in the approach to investigating such systems. 